\title{
Identification of nonserotypeable Shigella spp. using genome sequencing: a step forward
}

\begin{abstract}
Aim: Sequencing technology has replaced conventional methods in identifying and characterizing bacterial pathogens. We characterized 23 nonserotypeable Shigella that biochemically resembled Shigella spp. using whole genome sequencing. Materials \& methods: Genome sequences were analyzed using online tools based on $16 \mathrm{~S}$ rRNA, k-mer, gyrB sequences and analysis of O-antigen arrangement was done using PATRIC database for species identification. Sequence types, plasmid types, antimicrobial resistance and virulence genes were also investigated. Results: The SpeciesFinder using $16 \mathrm{~S}$ rRNA sequences identified only $74 \%$ of the isolates, whereas KmerFinder and gyrB sequence analysis identified $100 \%$ of the isolates to its species level. Antimicrobial resistance, virulence and plasmid incompatibility groups were identified in all the isolates. Sequence types were determined. Conclusion: This study shows that whole genome sequencing approach for Shigella O-antigen analysis has greater discriminative power than other methods using different bioinformatics pipeline for identification of nonserotypeable Shigella.
\end{abstract}

Lay abstract: Shigellosis is one of the major health concerns in developing countries. Shigella spp. that is not serotypeable with available antisera is a hurdle in laboratory diagnosis. Sequencing technology has replaced conventional methods in identifying nonserotypeable Shigella. In this study, we characterized 23 nonserotypeable Shigella using whole genome sequences. Manual comparison of $\mathrm{O}$-antigen gene arrangement provided definitive results compared with other methods. Development of a database including whole genome sequencing of all known serotypes will help in easy identification of Shigella serotypes in the future.

First draft submitted: 24 May 2017; Accepted for publication: 30 May 2017; Published online: 7 July 2017

Keywords: IncF plasmid $\bullet$ k-mer $\bullet$ nonserotypeable Shigella $\bullet$ O-antigen gene cluster

- whole genome sequencing

Shigella species have been reported to be the major cause of human gastroenteritis in developing countries. This includes $S$. dysenteriae, S. flexneri, S. boydii and S. sonnei. The diarrheal disease caused by Shigella is highly contagious due to its low infectious dose. Identification of the bacterial species in clinical specimens is crucial for choosing optimal treatment and for infection control measures. Identification of Shigella spp. by biochemical characteristics is suboptimal and requires serotyping for complete identification [1]. The variability of $\mathrm{O}$ antigen provides the major basis for serotyping of many Gramnegative bacteria, and it is the only antigen used for serotyping of Shigella as they lack $\mathrm{H}$ and $\mathrm{K}$ antigens [2]. Rarely, the genus Shigella is difficult to serotype with the available Shigella polyvalent and monovalent antisera, which could be due to morphological transi-
Muthuirulandi Sethuvel Dhiviya Prabaa', Devanga Ragupathi Naveen Kumar', Inbanathan Francis Yesurajan', Shalini Anandan', Walia Kamini² \& Veeraraghavan Balaji ${ }^{*, 1}$ 'Department of Clinical Microbiology, Christian Medical College, Vellore 632 004, India ${ }^{2}$ Division of Epidemiology \& Communicable Diseases, Indian Council of Medical Research, New Delhi 110029 , India

*Author for correspondence: Tel.: +919442210555 vbalaji@cmcvellore.ac.in 
tion from smooth to rough forms. Rough isolates do not produce $\mathrm{O}$-antigen (which is responsible for serological diversity) due to mutations in one or more of the multiple genes controlling $\mathrm{O}$-antigen synthesis and polymerization [3]. Further, the modification in the $S$. flexneri O-antigen backbone except serotype 6 gives rise to different serotypes due to the addition of glucosyl, O-acetyl or phosphoethanolamine groups to one or more sugars [4].

Several studies have reported that Shigella spp. and other closely related species (such as enteroinvasive $E$. coli) share a similar pathogenic mechanism, yet it is essential to discriminate due to their clinical relevance and for outbreak responses. This close relatedness makes biochemical- and serological-based identification difficult. Although various molecular methods like restriction fragment length polymorphism targeting the $r f b$ and $f l i C$ gene, ribotyping, PFGE (pulse field gel electrophoresis), MALDI-TOF MS and PCR-based methods including ERIC (Enterobacterial Repetitive Intergenic Consensus)-PCR have been proposed in the past years, the discrimination between the species is still challenging $[5,6]$.

Recently, a whole-genome sequence (WGS) based analysis using publicly available bioinformatics tool was found to replace traditional methods. WGS analysis shows better discrimination between closely related species and can provide clinically relevant information [7]. The report by Chattway et al., showed that the $\mathrm{k}$-mer-based identification approach on whole genome data effectively differentiated Shigella from E. coli and provided information on phylogenetic relationship [8].

In this study, we studied the whole genome of 23 nontypeable Shigella isolates by various in-silico methods. Multiple analyses using organism-specific bioinformatics pipeline were performed to resolve the identification difficulty. Additionally, the isolates were investigated for the presence of antimicrobial resistance (AMR) genes, plasmids. Sequence types were also determined.

\section{Materials \& methods}

\section{Bacterial strains}

Seven hundred and sixty four Shigella isolates were collected during the years 2011-2015 at Christian Medical College, Vellore, India. All the strains were isolated from stool specimens of patients with gastroenteritis. Individual isolates were characterized using standard biochemical tests [9]. Serologic identification was done by slide agglutination test using polyvalent somatic (O) antigen grouping sera, followed by monovalent antisera (Denka, Seiken, Japan) for Shigella specific serotype identification. Twenty-three Shigella isolates out of 48 Shigella spp. that were identified to be non- agglutinable with either poly- or monovalent Shigella antisera were randomly selected and included in the study for further characterization.

\section{Antimicrobial susceptibility testing}

All the isolates were tested for antimicrobial susceptibility against the following antibiotics: ampicillin (10 $\mu \mathrm{g})$, trimethoprim/sulfamethoxazole $(1.25 / 23.75 \mu \mathrm{g})$, nalidixic acid $(30 \mu \mathrm{g})$, norfloxacin $(10 \mu \mathrm{g})$, cefotaxime $(30 \mu \mathrm{g})$ and cefixime $(5 \mu \mathrm{g})$ by disc diffusion method. Results were interpreted using Clinical and Laboratory Standards Institute Guidelines 2015 [10]. Quality control strains used were: E. coli ATCC 35218 for ampicillin and E. coli ATCC 25922 for rest of the antibiotics.

\section{Next generation sequencing}

The WGS for the study strains were performed using Ion Torrent (PGM, Life Technologies, CA, USA) with 400-bp read chemistry (Life Technologies). Library preparations were performed according to manufacturer's instructions using (Ion Plus Fragment Library Kit; Life Technologies). Genomic library was purified using AMPure beads, and concentrations were determined using the Qubit 3.0 fluorimeter (Invitrogen, Merelbeke, Belgium). Emulsion PCR was performed on pooled libraries (Ion One Touch Hi-Q 400 Template Kit v2 DL Kit; Life Technologies), and templatepositive Ion Sphere particles were enriched using Dynabeads Myone streptavidin C1 beads. Finally pooled samples were loaded on an Ion 318 chip for sequencing.

\section{Assembly \& annotation}

The generated whole genome data were assembled de novo using AssemblerSPAdes v.5.0.0.0 embedded in Torrent suite server v.5.0.4. The genome sequence was annotated using PATRIC, the bacterial bioinformatics database and analysis resource (www.patricbrc.org), and the NCBI Prokaryotic Genome Automatic Annotation Pipeline (www.ncbi.nlm.nih.gov/genomes/ static/Pipeline.html) [11].

\section{Downstream genome analysis}

The whole genome data were analyzed using open access tools at Center for Genomic Epidemiology (CGE) web-based server. Sequence types for the study isolates were determined using multilocus sequence typing 1.8 tool (MLST 1.8) (https:// cge.cbs.dtu.dk//services/MLST/) [12], AMR and virulence genes were identified using ResFinder 2.1 (https://cge.cbs.dtu.dk//services/ResFinder/) [13] and VirulenceFinder 1.5 (https://cge.cbs.dtu.dk// services/VirulenceFinder/) [14] with the $90 \%$ threshold for identity and with $60 \%$ of minimum length 
coverage. Presence of plasmids were analyzed using PlasmidFinder 1.3 (https://cge.cbs.dtu.dk//services/ PlasmidFinder/) [15] with $95 \%$ threshold for identity.

\section{WGS analysis for identification of nonserotypeable Shigella \\ Species identification using $16 \mathrm{~S}$ rRNA sequence analysis}

SpeciesFinder 1.0 predicts species based on their 16S rRNA gene, where the assembled genome sequences will be aligned against the16S rRNA sequences from the database using the default BLAST algorithm (https://cge.cbs.dtu.dk/services/SpeciesFinder/) [16]. The best BLAST hit was chosen based on the query coverage, \% identity, number of mismatches and number of gaps in the alignments.

\section{k-mer-based species identification}

This method finds the unique k-mers in the input sequence and predicts species based on the number of overlapping k-mers, that is, 16-mers, between the query genome and genomes in a reference database. The prediction was made at which it has the highest number of 16-mers in common despite of position. The program ran with the 'winner takes it all' scoring method (https://cge.cbs.dtu.dk/services/KmerFinder/) [7].

\section{gyrB sequence based species identification}

Shigella genomes were analyzed for $g y r \mathrm{~B}$ gene sequences to classify closely related species. The query gyrB sequences obtained from WGS were manually BLAST matched using NCBI pipeline [17].

\section{Manual serotype identification using $\mathrm{O}$-antigen} gene cluster

The number of genes in $\mathrm{O}$-antigen clusters varies and strains of different serotypes can show completely different gene sets. The sequential arrangements of $\mathrm{O}$-antigen genes which are usually bordered by galF and gnd genes were compared manually with those available as Shigella WGS database in the PATRIC database [18].

\section{Phylogenetic analysis}

Phylogenetic analysis was performed for $g y r \mathrm{~B}$ sequences of the isolates on the Phylogeny.fr platform. Sequences were aligned with MUSCLE (v3.8.31) with the default settings. After alignment, gaps and poorly aligned sequences were removed with Gblocks (v0.91b) using the following parameters: minimum length of a block after gap cleaning: ten, no gap positions were allowed in the final alignment, all segments with contiguous nonconserved positions bigger than eight were rejected and minimum number of sequences for a flank position: $85 \%$.

The phylogenetic tree was reconstructed using the maximum likelihood method implemented in the PhyML program (v3.1/3.0 aLRT). The HKY85 substitution model was selected assuming an estimated proportion of invariant sites (of 0.910) and four $\gamma$-distributed rate categories to account for rate heterogeneity across sites. The $\gamma$-shape parameter was estimated directly from the data $(\gamma=95.586)$. Reliability for internal branch was assessed using the aLRT test (SH-Like). Graphical representation and edition of the phylogenetic tree were performed with TreeDyn (v198.3) [19,20].

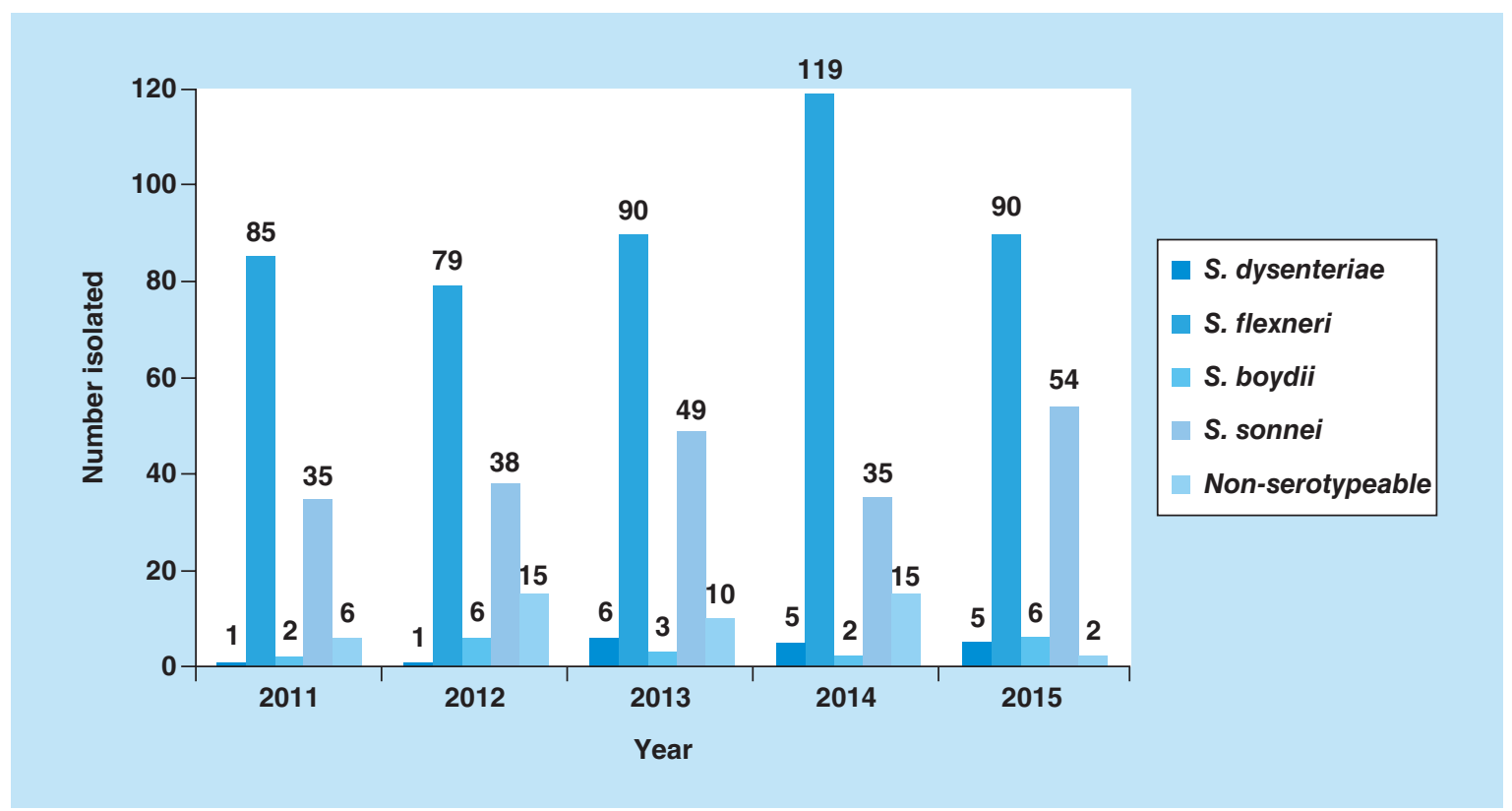

Figure 1. Serogroup distribution of Shigella from feces specimens over 5 years (2011-2015). 


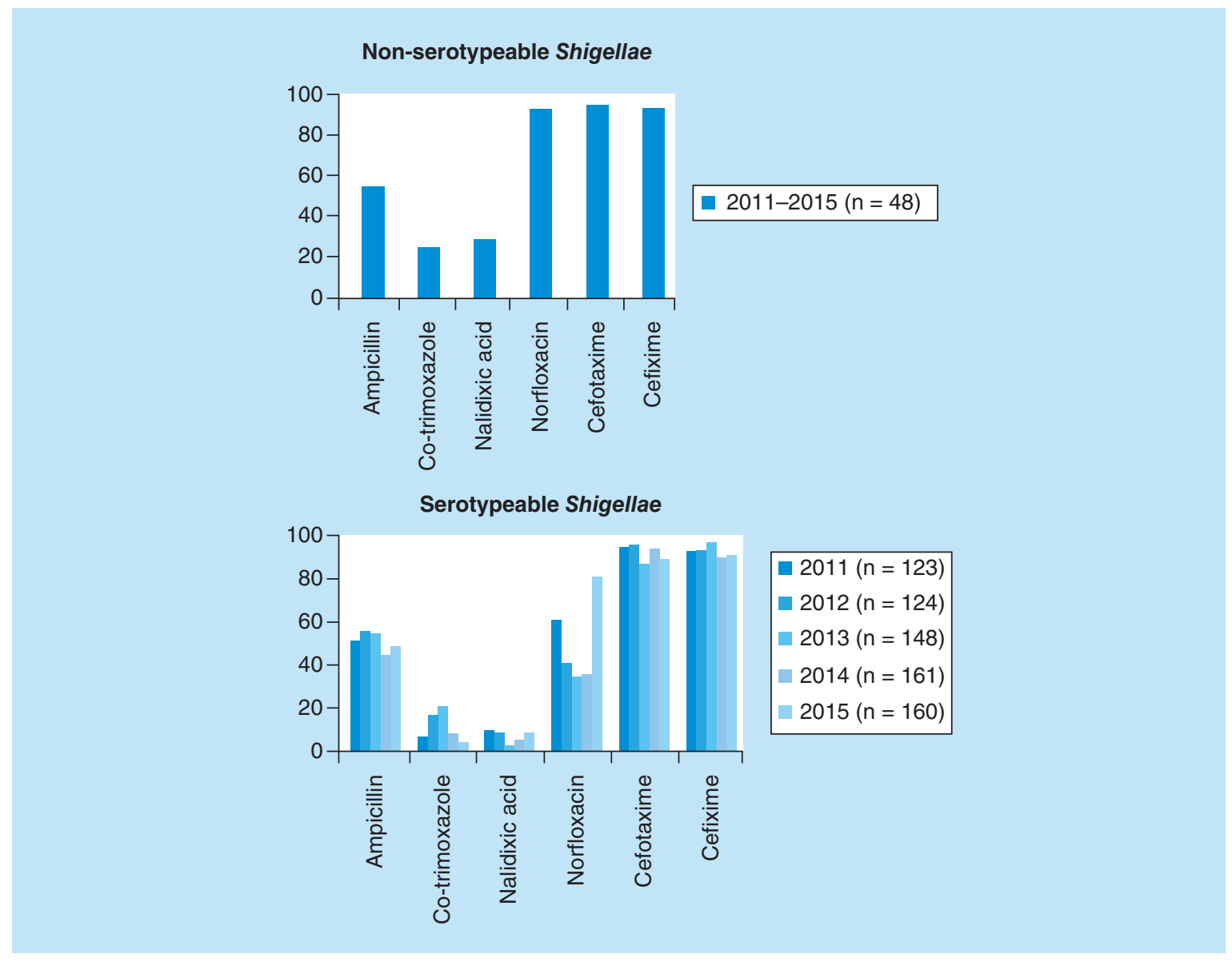

Figure 2. Antimicrobial susceptibility trend of serotypeable and nonserotypeable Shigella spp. over 5 years (2011-2015).

\section{Results}

From 2011 to 2015, a total of 716 Shigella species with known serotypes were isolated with $S$. flexneri the predominant serogroup followed by $S$. sonnei (Figure 1).

\section{AMR profile}

Among the selected isolates $48 \%$ were found to be multidrug resistant. The isolates showed $69 \%$ resistance to nalidixic acid followed by trimethoprim/sulfamethoxazole $66 \%$, ampicillin $52 \%$, cefotaxime $17 \%$, norfloxacin $13 \%$ and cefixime $4 \%$, respectively. The antimicrobial susceptibility trends of nonserotypeable and typeable Shigella spp. over the past 5 years are presented in Figure 2.

\section{Genome analysis of nonserotypeable Shigella \\ SpeciesFinder}

SpeciesFinder predicts the prokaryotic species based on the 16S rRNA gene similarity with the known reference sequence. This method identified eleven isolates (FC1, FC 2, FC6-FC9, FC14-FC17 and FC20) as S. boydii, three isolates (FC10-FC12) as S. flexneri but failed to identify the species for six isolates (FC3-FC5, FC13, FC18 and FC19). Further three isolates (FC21FC23) were identified as E. coli (Table 1).

\section{KmerFinder}

KmerFinder identified the isolates (FC1-FC9, FC13FC20) as $S$. boydii and isolates FC10, FC11 and FC12 as S. flexneri. The three isolates (FC21, 22 and 23) showed similar results as that of SpeciesFinder, by k-mer method and were identified as E. coli. (Table 1).

\section{GyrB sequence based species identification}

This method targets gyrB gene that encode the subunit B protein of DNA gyrase (topoisomerase type II protein). $g y r \mathrm{~B}$ gene sequences has greater divergence values between the closely related species. BLAST matching the query sequence resulted in identification of the species based on sequence similarity. The results of gyrB gene sequence analysis matches with that of KmerFinder as shown in Table 1. But this method identified seven isolates as $S$. boydii only with 
Table 1. Comparative analysis of SpeciesFinder, KmerFinder, gyrB sequence and O-antigen gene sequence based identification of nonserotypeable Shigella.

\begin{tabular}{|c|c|c|c|c|c|}
\hline \multirow[t]{2}{*}{ Isolate ID } & \multirow{2}{*}{$\begin{array}{l}\text { Manual analysis } \\
\text { using PATRIC }\end{array}$} & \multicolumn{2}{|c|}{ Center for Genomic Epidemiology } & \multirow{2}{*}{$\begin{array}{l}\text { BLAST } \\
\text { gyrB sequence } \\
\text { analysis }\end{array}$} & \multirow{2}{*}{$\begin{array}{l}\text { Sequence } \\
\text { types }\end{array}$} \\
\hline & & SpeciesFinder & KmerFinder & & \\
\hline FC1 & S. boydii & S. boydii & S. boydii & S. boydii & 145 \\
\hline $\mathrm{FC2}$ & S. boydii & S. boydii & S. boydii & S. boydii & 145 \\
\hline FC3 & S. boydii & Matching failed & S. boydii & S. boydii & 243 \\
\hline FC4 & S. boydii & Matching failed & S. boydii & S. boydii & 145 \\
\hline FC5 & S. boydii & Matching failed & S. boydii & S. boydii & 145 \\
\hline FC6 & S. boydii & S. boydii & S. boydii & S. boydii & 145 \\
\hline FC7 & S. boydii & S. boydii & S. boydii & S. boydii & 145 \\
\hline FC8 & S. boydii & S. boydii & S. boydii & S. boydii & 145 \\
\hline FC9 & S. boydii & S. boydii & S. boydii & S. boydii & 145 \\
\hline FC10 & S. flexneri $2 a$ & S. flexneri & S. flexneri & S. flexneri & 245 \\
\hline FC11 & S. flexneri 2a & S. flexneri & S. flexneri & S. flexneri & 245 \\
\hline FC12 & S. flexneri $2 a$ & S. flexneri & S. flexneri & S. flexneri & 245 \\
\hline $\mathrm{FC} 13$ & S. dysenteriae & Matching failed & S. boydii & S. boydiit & 148 \\
\hline FC14 & S. dysenteriae & S. boydii & S. boydii & S. boydii & 148 \\
\hline FC15 & S. dysenteriae & S. boydii & S. boydii & S. boydii ${ }^{\dagger}$ & 148 \\
\hline FC16 & S. dysenteriae & S. boydii & S. boydii & S. boydii ${ }^{\dagger}$ & 148 \\
\hline FC17 & S. dysenteriae & S. boydii & S. boydii & S. boydii & 148 \\
\hline FC18 & - & Matching failed & S. boydii & S. boydii & 243 \\
\hline FC19 & - & Matching failed & S. boydii & S. boydii ${ }^{\dagger}$ & 148 \\
\hline FC20 & - & S. boydii & S. boydii & S. boydii ${ }^{\dagger}$ & 148 \\
\hline FC21 & - & E. coli & E. coli & E. coli & 6199 \\
\hline $\mathrm{FC} 22$ & - & E. coli & E. coli & E. coli & 6199 \\
\hline $\mathrm{FC} 23$ & - & E. coli & E. coli & E. coli & 6270 \\
\hline
\end{tabular}

99\% identity to the reference genome available in the NCBI database.

Manual serotype identification by $\mathrm{O}$-antigen gene analysis

The genome sequence analysis revealed that the $\mathrm{O}$-antigen structure of the isolates $\mathrm{FC1}-\mathrm{FC} 9$ matched with the reference strain $S$. boydii in the database. Isolates FC10-FC12 were found to be $S$. flexneri 2a, and was concordant with all methods. In contrast, five isolates (FC13-FC17) had O-antigen structure similar to $S$. dysenteriae through manual comparison but was identified as $S$. boydii by other three methods. However, the O-antigen gene arrangement of five isolates (FC18-FC22) did not match with any of the reference Shigella sequence in the PATRIC database.

\section{MLST/AMR/virulence identification}

MLST reveals the sequence type of the isolates based on allelic profile. The sequence types of the isolates were determined using E. coli MLST database [23]. Shigella boydii isolates $(\mathrm{n}=9)$ were found to have two sequence types, ST145 and ST243 (Table 1). Five isolates identified as $S$. dysenteriae belong to ST148, and three S. flexneri isolates were identified as ST245.

Acquired AMR genes were identified in all except four isolates (FC4, 5, 11 and 23). The virulence genes responsible for Shigella pathogenesis are located in the chromosome and in the invasion (inv) plasmid. Multiple virulence factors were identified in 22 isolates using VirulenceFinder tool and are given in Table 2. Plasmid analysis revealed the presence of various incompatibility groups among the isolates, of which IncFII was the most common type $(\mathrm{n}=19)$. Five isolates $(\mathrm{FC} 4$, 
Table 2. Genomic characteristics of nonserotypeable Shigella $(n=23)$.

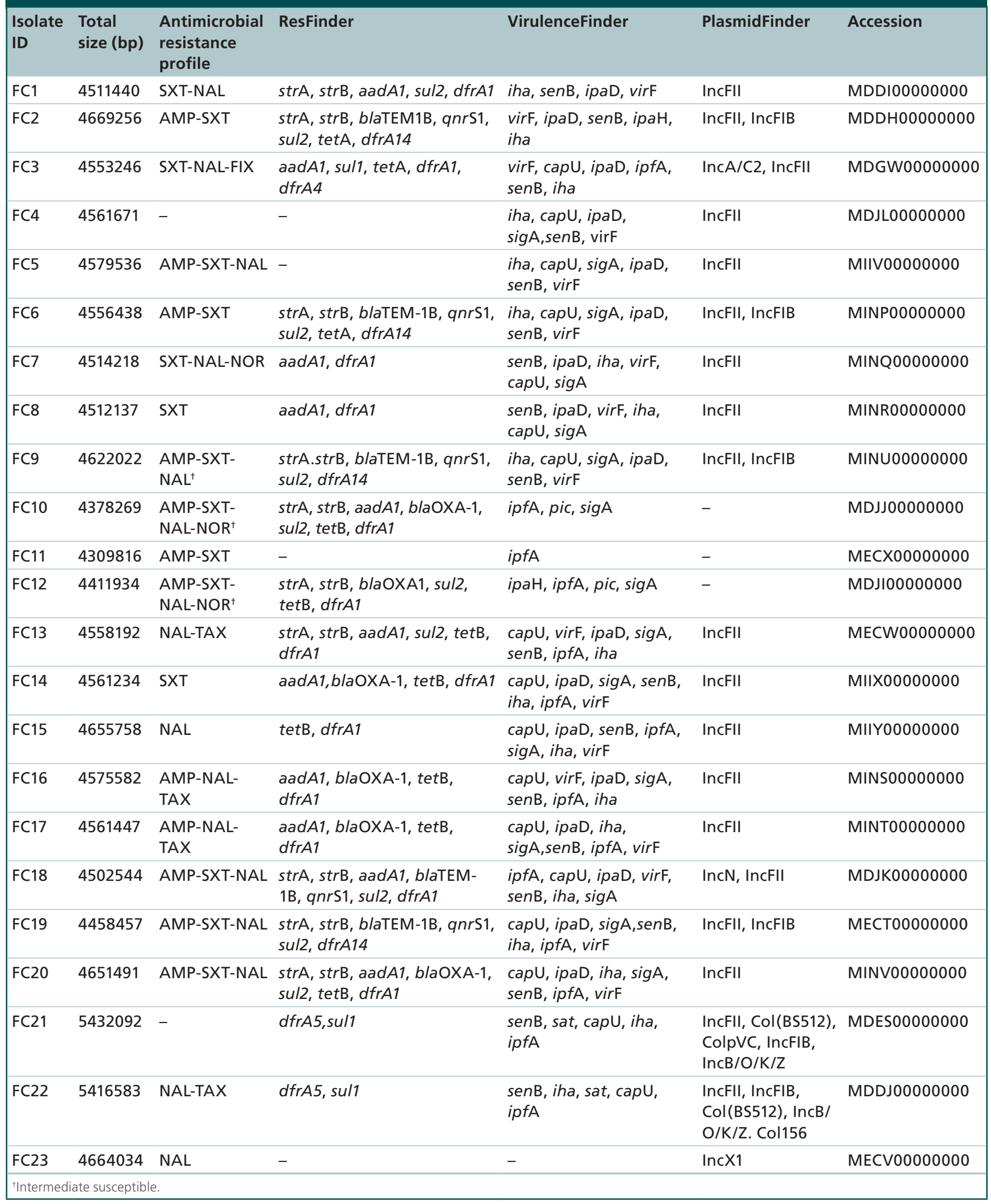


FC5, FC10, FC12 and FC23) were found to have either AMR genes or plasmids (Table 2) and one isolate (FC11) neither had any AMR genes nor plasmids.

\section{Phylogenetic analysis of gyrB gene}

Along with the study isolates, known gyr B sequence data of S. dysenteriae, S. boydii, S. flexneri and E. coli were included to deduce the relationship between isolates. Within these three strains, FC21, 22 and 23, were E. coli, while other strains were Shigella spp. which is related to their common ancestor E. coli. FC10, 11 and 12 form a branch and matches with $S$. flexneri, while others are related to $S$. boydii and formed a separate clade. In this clade, 8 strains (FC1, 2, 4, 5, 6, 7, 8 and 9) were found to be $S$. boydii, from which two branches were evolved. One branch had seven strains (FC13, 14, $15,16,17,19$ and 20) with $92 \%$ similarity with S. boydii and the other had two strains (FC3 and 18) with 95\% similarity with S. dysenteriae. The phylogenetic tree for the isolates based on their $\mathrm{gyr} \mathrm{B}$ gene sequences is shown in the Figure 3.

\section{Discussion}

Although Shigella and E. coli share common characteristics there is often a clinical or public health need to differentiate these pathogens as they have different entities in epidemiology and clinical medicine. The incidence of new variants of Shigella serotype which do not agglutinate with commercially available antisera is increasing [21]. Studies on molecular characterization of such isolates are very rare especially in developing countries with a lower resource setting. In one such study, research from Kolkata, India, characterized 13 provisional serovars of Shigella with respect to their AMR, plasmids, virulence genes and PFGE profiles [21].

Sequence types identified in the current study are reported earlier. Previous studies have shown that the sequence type ST245 was found in various $S$. flexneri serotypes (1a, 1b, 2a, 2b, 3a, 3b, 3c, 4a, 4b, 4c and X) [22]. ST243 was recognized in S. flexneri serotypes 6 and 6a, S. boydii 1, 3, 6, 8, 10 and 18 and $S$. dysenteriae 5 and 7. Several STs may be present within a serotype, therefore categorizing the serotypes based on the ST's is not possible [22].

Previous studies from Iran and other countries observed that the tetracycline resistance was seen in the majority of the clinical Shigella strains due to the development of intrinsic resistance to this antibiotic. Shahsavan et al., reported the MLST of multidrug resistant Shigella spp. and found that ampicillin resistance was frequently observed in ST245 and tetracycline susceptibility in S. flexneri ST145 strain. This suggests that isolates with different sequence types correspond to specific AMR patterns. Regular evaluation of ST and AMR data will help to treat shigellosis caused by resistant strains [23]. In this study, eight isolates identified as $S$. boydii belong to ST145. Novel sequence types were obtained for E. coli (ST6199 and ST6270) in this study and have not been reported earlier.

Genome annotation results showed more than 50-times coverage for all the isolates. The genome sequences were analyzed in-silico by different methods using various web-based tools for species identification. SpeciesFinder, which is based on the 16S rRNA gene, had shown lesser resolution than the other methods discussed in this study, which identified only 17 isolates to species level. This could be due to substantial intergene variation and the fact that $16 \mathrm{~S}$ rRNA gene corresponds to $0.1 \%$ of the coding part of a microbial genome (Table 3). Bacterial identification using $16 \mathrm{~S}$ rRNA sequencing was reported to have limitations such as its inability to distinguish atypical $E$. coli from Shigella spp. The sequence similarities of S. flexneri, S. sonnei and S. boydii with E. coli were reported to be 99.8, 99.9 and 99.7\%, respectively [24]. This shows that the species identification by $16 \mathrm{~S}$ rRNA sequences is not reliable and accurate.

KmerFinder identified all the isolates to species level. The method predicts the species based on the closest match to the isolate in the k-mer database. Among the tested isolates, FC17 and 12 isolates results were in concordance with the SpeciesFinder and PATRIC respectively.

The gyr B gene sequence analysis presented better results when compared with $16 \mathrm{~S}$ rRNA analysis; this is in accordance with the previous study, where $g y r \mathrm{~B}$ gene analysis was found to be effective in classifying closely related species [17]. The results obtained by this method matched with KmerFinder results. This can be explained as the rate of genetic divergence of $g y r \mathrm{~B}$ sequences differed greatly from $16 \mathrm{~S}$ rRNA sequence and have four- to tenfold increase in the length of branches between closely related species of Shigella and $E$. coli. The percent divergences of $E$. coli from $S$. sonnei, S. flexneri and S. boydii were 1.9, 2.3 and $2.0 \%$, respectively, indicating the reliability of $g y r \mathrm{~B}$ gene sequence analysis method than $16 S$ rRNA analysis [24]. However, this method identified few isolates with only 99\% identity and does not provide accurate (100\%) identification results.

Analysis of $\mathrm{O}$-antigen gene cluster arrangement using PATRIC revealed the isolates FC18-FC23 did not match with any known Shigella sequences in the PATRIC database, which could be due to limitations inherent to the database. However, the rest of the isolates showed similar results to those of SpeciesFinder, KmerFinder and gyr B sequence analysis methods. 
Five isolates (FC13-FC17) was identified as $S$. dysenteriae through manual comparison of $\mathrm{O}$-antigen using PATRIC but were misidentified as $S$. boydii by the other three methods. Traditionally, S. boydii and $S$. dysenteriae are physiologically similar but were differentiated biochemically by the mannitol test and these two species can be found within the same STs, which shows their close phylogenetic relationship.

Overall, SpeciesFinder, based on the 16S rRNA gene analysis, showed poor performance, which can identify only $74 \%$ of the isolates up to species level. KmerFinder and $g y r \mathrm{~B}$ sequence analysis both identified $100 \%$ of the isolates to the species level despite its accuracy.

Occasionally, O-antigen modification within the species affects the serotyping assay and makes the strain untypeable. The modification is widely seen in S. flexneri serotypes (1a, 1b, 2a, 5a and $\mathrm{Y}$ ) as these have bacteriophage-mediated serotype conversion [4]. Consequently the laboratory identification of Shigella by agglutination method becomes difficult with commercially available polyvalent-antisera and may not cover all possible epitopes of the Shigella $\mathrm{O}$-antigen. Another problem is due to high genetic similarity between $S h i-$ gella and E. coli and in fact that majority of Shigella $\mathrm{O}$ antigens cross-react serologically with some strains of E. coli $\mathrm{O}$ antigen [2]. It was found that cross-reacting strains of Shigella spp. and E. coli may express shared lipopolysaccharide epitopes yet their lipopolysaccharide structures are not identical [25]. Therefore, differentiation between Shigella and cross-reacting enteroinvasive $E$. coli strains are often difficult as both show similar biochemical traits and can cause dysentery using the same mode of invasion [26].

In contrast to the earlier study by Dutta et al. [21] who demonstrated that multidrug resistance was reported rarely in the provisional serovars of Shigella isolates, most of our study isolates were found resistant to first-line antibiotics like ampicillin, trimethoprim/ sulfamethoxazole and nalidixic acid but were highly susceptible to second-generation quinolone (norfloxacin) and third-generation cephalosporin (cefotaxime and cefixime). The nontypeable Shigella isolates from our setting over the last 5 years showed susceptibility of

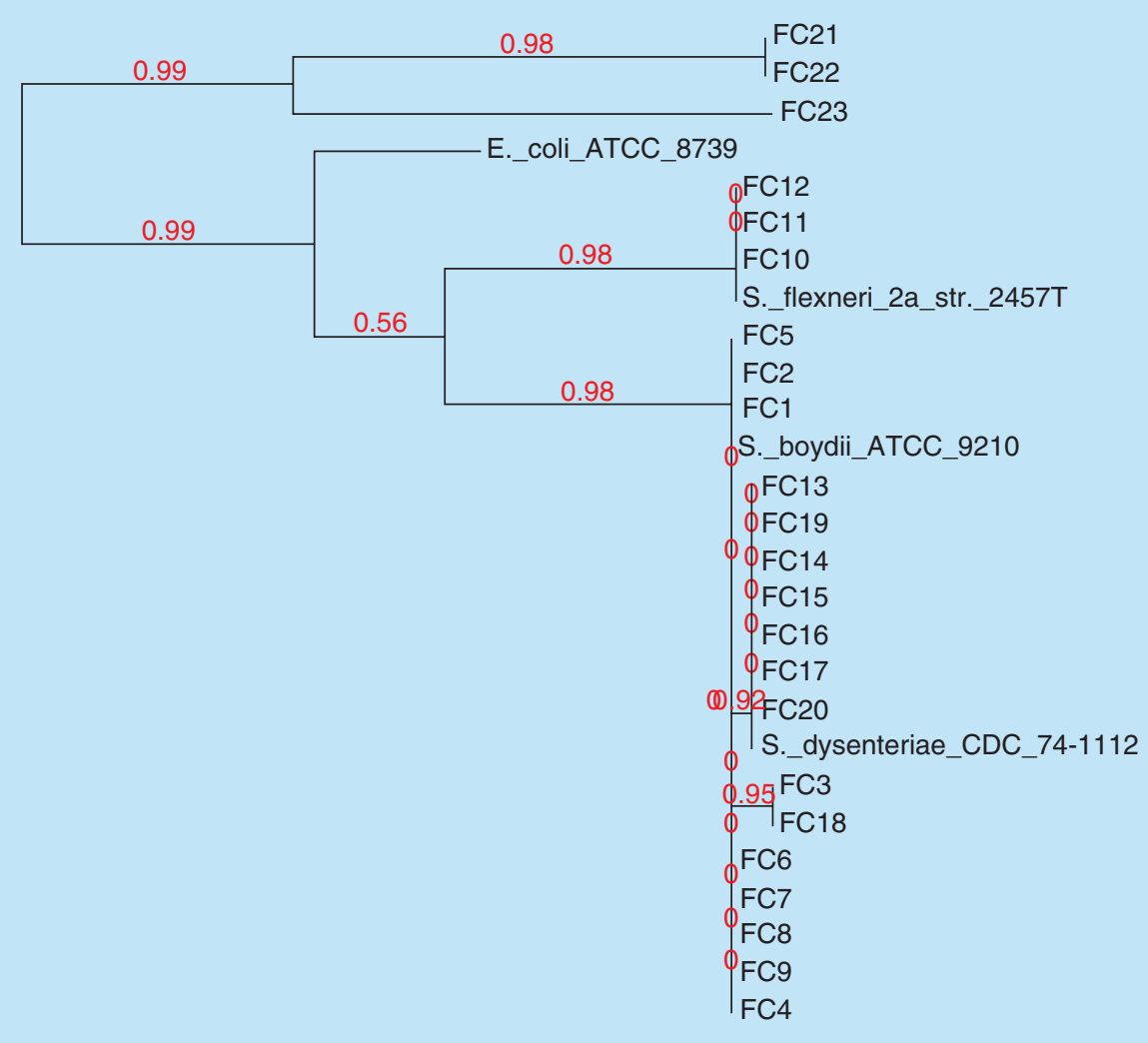

0.005

Figure 3. Phylogenetic tree was constructed using maximum likelihood method of nucleotide sequences of gyrB genes for the study isolates. 
Table 3. 16S rRNA sequence similarity within Shigella species.

\begin{tabular}{|llllll|}
\hline $\begin{array}{l}\text { Organism } \\
\text { S. dysenteriae }\end{array}$ & S. dysenteriae & S. boydii & S. sonnei & S. flexneri & S. flexneri 5a \\
\hline S. boydii & 98.72 & 100 & & & \\
\hline S. sonnei & 98.79 & 99.4 & 100 & & \\
\hline S. flexneri & 99.06 & 99.66 & 99.73 & 100 & \\
\hline S. flexneri 5a & 98.86 & 99.47 & 99.68 & 99.8 & 100 \\
\hline
\end{tabular}

around $90 \%$ to norfloxacin, cefotaxime and cefixime in contrast to typeable Shigella isolates but had similar resistance profile for ampicillin. Serotypeable isolates were highly resistant to co-trimoxazole and nalidixic acid compared with nontypeable isolates.

Further, the study isolates were found to harbor AMR genes through sequence analysis and the results correlate with the phenotypic resistance profile except for three isolates (FC5, FC11 and FC23). One isolate (FC21) does not show any resistance phenotypically but had AMR genes.

Molecular typing of nonserotypeable Shigella is important as this species represents the major causes of bacterial diarrhea in developing countries. This study shows that some limitations such as inability to distinguish closely related species were observed with the $16 \mathrm{~S}$ rRNA sequence method, but this can be overcome by the k-mer approach and $g y r \mathrm{~B}$ gene analysis. However, the manual comparison of $\mathrm{O}$-antigen gene arrangement provided considerable and more definitive results than the k-mer and $g y r \mathrm{~B}$ method in identifying the species although it could not identify a few isolates due to the lack of genome sequences of all Shigella serotypes in the database. This study underlines the need for genome sequences of all known Shigella serotypes reported till date, for detailed understanding of the species with enhanced resolution.

\section{Limitations}

A reference database including $\mathrm{O}$-antigen sequences of all known serotypes of Shigella spp. was not available. Manual comparison of O-antigen arrangements is error prone; however, utmost care was taken to avoid such errors.

\section{Conclusion \& future perspective}

The O-antigen determination of clinical Shigella isolates is essential for diagnostic and epidemiologic purpose. Knowledge on the distribution of different Shigella serotypes remains important as humans demonstrate only serotype-specific immunity. Therefore, the characterization of isolates using WGS could provide better results and have greater discriminative power compared with other commonly used methods. Development of a database including WGS of all known Shigella serotypes is required for future identification and comparison of nontypeable Shigella. Also, an SNP-based phylogenetic analysis will be a supporting evidence for Shigella serotype diversification.

\section{Acknowledgements}

The authors gratefully acknowledge the Institutional Review Board for approving the study and the department of Clinical Microbiology for providing lab space and facilities.

Financial \& competing interests disclosure

The study was funded by the Indian Council of Medical Research (ICMR), New Delhi (Ref. no: AMR/TF/55/13ECDIl dated 23 October 2013). The authors have no other relevant affiliations or financial involvement with any organization or entity with a financial interest in or financial conflict with the subject matter or materials discussed in the manuscript apart from those disclosed.

No writing assistance was utilized in the production of this manuscript.

\section{Ethical conduct of research}

The authors state that they have obtained appropriate institutional review board approval or have followed the principles outlined in the Declaration of Helsinki for all human or animal experimental investigations. In addition, for investigations involving human subjects, informed consent has been obtained from the participants involved.

\section{Open access}

This work is licensed under the Creative Commons Attribution 4.0 License. To view a copy of this license, visit http://creativecommons.org/licenses/by/4.0/

\section{Authors' contributions}

$\checkmark$ Balaji and S Anandan conceptualized the study. MSD Prabaa and DRN Kumar analyzed, interpreted data and wrote the manuscript. MSD Prabaa and FY Inbanathan carried out bench work, generated data. V Balaji, K Walia and DRN Kumar critically revised and approved the manuscript. All authors read and approved the manuscript. 
Summary points

\section{Background}

- Identification of the bacterial species in clinical specimens is crucial for choosing optimal treatment and for infection control measures.

- The discrimination between closely related species is still challenging. This close relatedness makes the biochemical and serological based identification difficult.

- Recently, whole genome sequencing technology has replaced the conventional methods in identifying and characterizing the bacterial pathogens.

- Here, we studied the whole genome of 23 nonserotypeable Shigella isolates to resolve the identification difficulty.

- Overall, SpeciesFinder, based on the 16S rRNA gene, had the poorest performance, which identifies only $74 \%$ of the isolates up to its species level.

- Whereas KmerFinder and gyrB sequence analysis, both had the highest performance and identify $100 \%$ of the isolates to the species level.

- The manual comparison of O-antigen gene arrangement was considered to be the reliable method for identifying nonserotypeable Shigella.

\section{Conclusion}

- Whole genome sequencing was found to have greater discriminative power compared with other commonly used methods.

- Also knowledge on the distribution of different Shigella serotypes remains important as humans demonstrate serotype specific immunity.

\section{References}

Papers of special note have been highlighted as: • of interest; •• of considerable interest

1 Grimont F, Lejay-Collin M, Talukder KA et al. Identification of a group of Shigella-like isolates as Shigella boydii 20. J. Med. Microbiol. 56, 749-754 (2007).

2 Liu B, Knirel YA, Feng L et al. Structure and genetics of Shigella $\mathrm{O}$ antigens. FEMS Microbiol. Rev. 34, 606 (2008).

3 Coimbra RS, Grimont F, Grimont PAD. Identification of Shigella serotypes by restriction of amplified $\mathrm{O}$-antigen gene cluster. Res. Microbiol. 150, 543-553 (1999).

- The purpose of this work was to identify an $\mathrm{O}$ antigen pattern for serotype identification without the use of antisera. This is the first study where the authors developed a discriminating method called $\mathrm{O}$-antigen gene cluster amplification for Shigella identification and typing. Also a database was built with the Taxotron package allowing automated identification of clinical Shigella isolates to all known serotypes.

4 Jakhetia R, Marri A, Stahle J, Widmalm G, Verma NK. Serotype-conversion in Shigella flexneri: identification of a novel bacteriophage, Sf101, from a serotype 7. BMC Genomics 15, 742 (2014).

5 Muthuirulandi Sethuvel DP, Devanga Ragupathi NK, Anandan S, Walia K, Veeraraghavan B. Molecular diagnosis of non-serotypeable Shigella spp.: problems and prospects. J. Med. Microbiol. 66, 255-257 (2017).

6 Pettengill EA, Pettengill JB, Binet R. Phylogenetic analyses of Shigella and enteroinvasive Escherichia coli for the identification of molecular epidemiological markers: wholegenome comparative analysis does not support distinct genera designation. Front. Microbiol. 6, 1573 (2016).
- Shows that SNP markers offer more discriminatory power to molecular epidemiological typing methods involving these bacterial pathogens.

7 Hasman H, Saputra D, Sicheritz-Ponten T et al. Rapid whole-genome sequencing for detection and characterization of microorganisms directly from clinical samples. J. Clin. Microbiol. 52, 139-146 (2014).

8 Chattaway MA, Schaefer U, Tewolde R, Dallman TJ, Jenkins C. Identification of Escherichia coli and Shigella species from whole genome sequences. J. Clin. Microbiol. doi:10.1128/JCM.01790-16 (2016) (Epub ahead of print).

- Showed that Shigella can be differentiated from E. coli and accurately identified to the species level from whole genome sequencing data by use of a k-mer-based approach.

9 Koneman EW, Allen SD, Janda WM, Schreckenberger PC, Winn WC. Colour Atlas and Textbook of Diagnostic Microbiology (5th Edition). Lippincott, NY, USA (1997).

10 CLSI, Clinical and Laboratory Standards Institute. Performance Standards for Antimicrobial Susceptibility Testing; Twenty-fourth Informational Supplement M100-S25. Clinical and Laboratory Standards Institute, MI, USA (2015).

11 Tatusova T, DiCuccio M, Badretdin A et al. NCBI prokaryotic genome annotation pipeline. Nucleic Acids Res. 44, 6614-6624 (2016).

12 Larsen MV, Cosentino S, Rasmussen S et al. Multilocus sequence typing of total genome sequenced bacteria. J. Clin. Micobiol. 50, 1355-1361 (2012).

13 Zankari E, Hasman $\mathrm{H}$, Cosentino $\mathrm{S}$ et al. Identification of acquired antimicrobial resistance genes. J. Antimicrob. Chemother. 67, 2640-2644 (2012).

14 Joensen KG, Scheutz F, Lund O et al. Real-time wholegenome sequencing for routine typing, surveillance, and outbreak detection of verotoxigenic Escherichia coli. J. Clin. Micobiol. 52, 1501-1510 (2014). 
attoli A, Zankari E, Garcia-Fernandez A et al. Plasmid Finder and pMLST: in silico detection and typing of plasmids. Antimicrob. Agents Chemother. 58, 3895-3903 (2014). Benchmarking of methods for genomic taxonomy. J. Clin. Microbiol. 52, 1529-1539 (2014).

17 Nochi Z, Sahebekhtiari N, Kharaziha P et al. Comparison of $16 \mathrm{~S}$ rRNA, 23S rRNA and gyrB genes sequences in phylogenetic relationships of Shigella isolates from Iran. Ann. Microbiol. 59, 615 (2009).

- Determines the phylogenetic relationship of Shigella spp. using $16 S$ rRNA, 23S rRNA and gyrB gene sequences.

18 Wattam AR, Abraham D, Dalay O et al. PATRIC, the bacterial bioinformatics database and analysis resource. $\mathrm{Nucl}$. Acids Res. 42, D581-D591 (2014).

www.patricbrc.org

19 Dereeper A, Guignon V, Blanc G et al. Phylogeny.fr: robust phylogenetic analysis for the non-specialist. Nucleic Acids Res. 36, W465-W469 (2008).

20 Dereeper A, Audic S, Claverie JM, Blanc G. Blast-explorer helps you building datasets for phylogenetic analysis. $B M C$ Evol. Biol. 10, 8 (2010).

21 Dutta S, Jain P, Nandy S, Matsushita S, Yoshida S. Molecular characterization of serologically atypical provisional serovars of Shigella isolates from Kolkata, India. J. Med. Microbiol. 63, 1696-1703 (2014).
- The first report in India which characterizes the isolates that biochemically resemble Shigella species but that does not belong to any of the recognized $\mathrm{O}$ serogroups with respect to their antimicrobial resistance profiles, plasmid profiles, virulence genes profiles and PFGE profiles.

22 Choi SY, Jeon YS, Lee JH et al. Multilocus sequence typing analysis of Shigella flexneri isolates collected in Asian countries. J. Med. Microbiol. 56, 1460-1466 (2007).

23 Shahsavan S, Nobakht M, Rastegar-Lari A, Owlia P, Bakhshi B. Multi-locus sequence type analysis of Shigella spp. isolates from Tehran, Iran. Iran J. Microbiol. 8, 298-306 (2016).

-. Provides data on the Shigella STs for better understanding of distribution and dominant sequence types in the region.

24 Fukushima M, Kakinuma K, and Kawaguchi R. Phylogenetic analysis of Salmonella, Shigella, and Escherichia coli strains on the basis of the gyrB gene sequence. J. Clin. Microbiol. 40, 2779-2785 (2002).

25 Chart H, Daniel RMA, Cheasty T. The expression of lipopolysaccharide by strains of Shigella dysenteriae, Shigella flexneri and Shigella boydii and their cross-reacting strains of Escherichia coli. FEMS Microbiol. Lett. 292, 21-26 (2009).

- The first study to describe the SDS-PAGE profiles of Shigella and E. coli lipopolysaccharide.

26 Ud-Din A, Wahid S. Relationship among Shigella spp. and enteroinvasive Escherichia coli (EIEC) and their differentiation. Braz. J. Microbiol. 45, 1131-1138 (2015). 